\title{
Adaptação cultural e validação de conteúdo do Cornell Musculoskeletal Discomfort Questionnaire (CMDQ) para o Brasil
}

\author{
Cultural adaptation and content validation of the Cornell Musculoskeletal Discomfort \\ Questionnaire (CMDQ) for Brazil \\ Adaptación cultural y validación de contenido del Cornell Musculoskeletal Discomfort Questionnaire \\ (CMDQ) para Brasil
}

Recebido: 09/09/2021 | Revisado: 22/09/2021 | Aceito: 24/09/2021 | Publicado: 25/09/2021

\author{
Joseph Daniel Alves Aleixo \\ ORCID: https://orcid.org/0000-0001-5391-6715 \\ Universidade Guarulhos, Brasil \\ E-mail: josephaleixo@gmail.com \\ Meline Rossetto Kron-Rodrigues \\ ORCID: https://orcid.org/0000-0003-2174-268X \\ Universidade Guarulhos, Brasil \\ E-mail: me_kron@hotmail.com \\ Alfredo Almeida Pina-Oliveira \\ ORCID: https://orcid.org/0000-0002-1777-4673 \\ Centro Universitário Campo Limpo Paulista, Brasil \\ E-mail: alfredo.oliveira@faccamp.br \\ Noélle de Oliveira Freitas \\ ORCID: https://orcid.org/0000-0001-6742-5247 \\ Universidade Guarulhos, Brasil \\ E-mail: nooliveirafreitas@gmail.com
}

\begin{abstract}
Resumo
Objetivo: Adaptar culturalmente o Cornell Musculoskeletal Discomfort Questionnaire (CMDQ) para o português do Brasil e avaliar a validade de conteúdo. Métodos: Estudo metodológico. Para adaptação cultural do CMDQ foram percorridas as cinco etapas propostas pela literatura. Para análise dos dados oriundos da avaliação do comitê de especialistas foi calculado o índice de validade de conteúdo (IVC) e o Coeficiente de Validade de Conteúdo por item (CVCi) sendo considerado valores acima de 0,80 e 0,78 , respectivamente. Resultados: O CMDQ foi submetido a tradução para o português por dois tradutores independentes. A partir das duas versões traduzidas foi desenvolvida uma versão consensual a qual foi submetida a retrotradução. As versões traduzidas, a versão consensual em português e as versões retrotraduzidas foram submetidas a avaliação por um comitê composto por cinco especialistas. Após a análise do comitê, o IVC total foi de 0,78 . Sete itens apresentaram CVCi abaixo de 0,78 (variação de 0,68 a 0,76). Sendo assim, os pesquisadores avaliaram os itens que apresentaram baixo valor de CVCi. Para a avaliação, consideraram-se todas as recomendações do comitê e alteraram-se todos os itens indicados pelos especialistas. Todas as alterações foram realizadas na versão retrotraduzida do CMDQ e reapresentada ao autor original para a aprovação da versão final. Conclusões: A etapa adaptação cultural do CMDQ para o Brasil foi finalizada obtendo-se resultados relevantes. O processo de validação do CMDQ está em etapa de finalização para que, assim, a versão final do instrumento possa ser administrada em pesquisas.
\end{abstract}

Palavras-chave: Ergonomia; Estudo de validação; Dor musculoesquelética; Transtornos traumáticos cumulativos.

\begin{abstract}
Objective: Culturally adapt the Cornell Musculoskeletal Discomfort Questionnaire (CMDQ) to Brazilian Portuguese and assess content validity. Method: Methodological study. The five steps proposed by the literature for cultural adaptation were adopted. To analyze the data from the expert committee evaluation, the content validity index (CVI) and the Content Validity Coefficient per item (CVCi) were calculated considering values above 0.80 and 0.78 , respectively. Results: The CMDQ was submitted for translation into Portuguese by two independent translators. Based on the two translated versions, a consensual version was developed which was subjected to a back-translation. The translated versions, the consensual version in Portuguese and the back-translated versions were submitted for evaluation by a committee composed of five experts. After analysis by the expert committee, the total CVI of the questionnaire was 0.78. Seven items had CVCi below 0.78 (range 0.68 to 0.76). For the evaluation, all the recommendations of the committee were considered, and all the items indicated by the experts were changed. The changes in the items were made according to expert recommendations. All changes were made in the back-translated
\end{abstract}


version of the CMDQ and resubmitted to the original author for approval of the final version. Conclusions: The cultural adaptation stage of the CMDQ for Brazil was completed with relevant results. The process of cultural adaptation and validation of the CMDQ is underway, the pre-test stage and, later, the validation stage will be carried out in research.

Keywords: Ergonomics; Validation study; Musculoskeletal pain; Cumulative trauma disorders.

\section{Resumen}

Objetivo: Adaptar culturalmente el Cornell Musculoskeletal Discomfort Questionnaire (CMDQ) al portugués brasileño y evaluar su validez de contenido. Método: Estudio metodológico. Para la adaptación cultural del CMDQ se siguieron los cinco pasos propuestos en la literatura. Para analizar los datos de la evaluación del comité de expertos se calcularon el índice de validez de contenido (IVC) y el coeficiente de validez de contenido por ítem (CVCi), considerando valores superiores a 0,80 y 0,78 , respectivamente. Resultados: El CMDQ fue traducido al portugués por dos traductores independientes. A partir de las dos versiones traducidas, se desarrolló una versión consensual y se sometió a una retrotraducción. Las versiones traducidas, la versión consensual en portugués y las versiones retrotraducidas fueron sometidas a evaluación por un comité compuesto por cinco expertos. Después del análisis del comité, el CVI total fue 0,78. Siete ítems tenían CVCi por debajo de 0,78 (rango de 0,68 a 0,76). Por lo tanto, los investigadores evaluaron los elementos que tenían un valor de CVCi bajo. Para la evaluación se consideraron todas las recomendaciones del comité y se modificaron todos los ítems indicados por los expertos. Los cambios en los elementos se realizaron de acuerdo con las recomendaciones de los expertos. Todos los cambios se realizaron en la versión retrotraducida del CMDQ y se volvieron a enviar al autor original para la aprobación de la versión final. Conclusiones: La etapa de adaptación cultural del CMDQ para Brasil se completó con resultados relevantes. Se está finalizando el proceso de validación de CMDQ para que se pueda gestionar la versión final del instrumento en investigación.

Palabras clave: Ergonomía; Estudio de validación; Dolor musculoesquelético; Trastornos de traumas acumulados.

\section{Introdução}

O termo ergo tem como referência a fundamentação de origem grega, que significa trabalho; já o termo nomos, correlaciona-se com as leis, também de origem grega, ou seja, de tal forma, são as leis que regem o trabalho. O termo ergonomia teve a sua primeira publicação em 1857 por Wojciech Jastrzebowski. Esse feito correlacionou uma abordagem científica que considerava os fatores humanos do indivíduo com a intenção de descrever e categorizar o trabalho humano com base na sua organização (Serranheira F et al., 2009).

A ergonomia baseia-se em uma abordagem centrada no ser humano. Tal antropocentricidade prentende resgatar uma melhor condição de trabalho com o intuito de se alcançar não apenas o aumento da produtividade, mas, sobretudo, melhorias na qualidade de vida dos trabalhadores (Monteiro, 2009).

Os postos de trabalho inadequados geram um impacto social negativo, podendo contribuir para o aparecimento de inúmeras patologias e distúrbios musculoesqueléticos, que podem implantar prejuízos biomecânicos e/ou funcionais. Dessarte, a ergonomia passa a ser um fator determinante para a luta pela saúde dos trabalhadores com foco na prevenção de acidentes e na promoção de melhorias nas condições de trabalho, por meio de uma adequação nas três esferas, física, cognitiva e organizacional (Abrahão, 2000).

Para a avaliação dos problemas musculoesqueléticos de trabalhadores, os profissionais da saúde e pesquisadores podem utilizar instrumentos e/ou questionários de avaliação próprios da saúde ocupacional. No Brasil, foi identificado o Questionário Nórdico de Sintomas Osteomusculares, o Nordic Musculoskeletal Questionnaire (NMQ) que tem como objetivo padronizar estudos sobre a avaliação de queixas musculoesqueléticas em uma abordagem ergonômica (Kuorinka et al., 1987).

O Questionário Nórdico de Sintomas Osteomusculares foi adaptado e validado para uso o no Brasil (Barros \& Alexandre NMC, 2003) e utilizado em pesquisas no país (Mota et al., 2014; Mota, Quadros Júnior, Munaro \& Vilela, 2014; Vitta, Bertaglia \& Padovani, 2008; Carvalho \& Alexandre, 2006). No entanto, o Questionário Nórdico de Sintomas Osteomusculares não engloba questões específicas relacionadas aos sintomas musculoesqueléticos como dor e desconforto e a abordagem de como esses sintomas interferem no trabalho. 
Nesse sentido, tal limitação instigou a busca na literatura científica e identificou-se o questionário Cornell Musculoskeletal Discomfort Question (CMDQ) (Hedge, Morimoto \& And McCrobie, 1999). O CMDQ foi desenvolvido no laboratório de fatores humanos na Universidade de Cornell, nos Estados Unidos (EUA). O CMDQ foi adaptado e validado para o idioma turco, persa, espanhol e alemão e identificada boas características psicométricas (Kreuzfeld, Seibt, Kumar M, Rieger \& Stoll, 2016; Carrasquero, 2015; Afifehzadeh-Kashani et al., 2011; Erdinc, Hot \& Ozkaya, 2011).

Sendo assim, o presente estudo tem como objetivo adaptar culturalmente o CMDQ para o português do Brasil e avaliar a validade de conteúdo.

\section{Método}

\subsection{Tipo de pesquisa}

Estudo metodológico. A autorização para adaptação e validação do CMDQ para o português do Brasil foi concedida pelo autor principal Dr. Alan Hedge.

\subsection{Procedimentos e amostra}

Para a adaptação cultural e validação de conteúdo do CMDQ foram percorridas as cinco etapas propostas na literatura (Beaton, Bombardier, Guillemin \& Ferraz, 2007; Beaton, Bombardier, Guillemin \& Ferraz, 2000; Guillemin, Bombardier \& Beaton, 1993): 1. Tradução do questionário para a língua portuguesa do Brasil; 2. Obtenção do primeiro consenso da versão em português; 3. Retrotradução (back-translation) do CMDQ; 4. Avaliação pelo comitê de especialistas e 5. Pré-teste.

A tradução do CMDQ foi realizada por dois tradutores independentes, nativos no idioma alvo (português do Brasil) e com conhecimento da língua e cultura inglesa. Um dos tradutores foi informado sobre o que o CMDQ avalia (Beaton et al., 2007; Beaton et al., 2000; Guillemin et al., 1993). Os tradutores desta etapa foram denominados tradutor 1 e tradutor 2 . As versões traduzidas oriundas desta etapa foram denominadas: Versão Tradutor 1 (VT1) e Versão Tradutor 2 (VT2).

Os dois tradutores realizaram uma avaliação das duas versões traduzidas comparando com a versão original. A avaliação foi realizada por meio de uma escala tipo Likert que varia de 1 a 4 , sendo 1 = item não relevante ou não representativo, $2=$ item necessita de grande revisão para ser representativo; $3=$ item necessita de pequena revisão para ser representativo; 4 = item relevante ou representativo (Rubio, Berg-Weger, Tebb, Lee \& Rauch, 2003; Lynn, 1986). O resultado da avaliação de cada tradutor foi analisado pelos pesquisadores do estudo os quais a partir desta avaliação construíram a Versão Consensual em Português 1 do CMDQ (VCP1).

As versões traduzidas, VT1 e VT2, foram submetidas a retrotradução, tradução para a língua materna do instrumento, por dois tradutores independentes, diferentes da etapa de tradução (Beaton et al., 2007; Beaton et al., 2000; Guillemin et al., 1993). Um tradutor era bilíngue, nativo no idioma alvo (português do Brasil) e com conhecimento da língua e cultura inglesa. $\mathrm{O}$ outro tradutor era bilíngue e nativo da língua materna do questionário (inglês) e com conhecimento da língua portuguesa e da cultura brasileira. Nesta etapa, os tradutores não tiveram conhecimento sobre os objetivos do CMDQ. Os tradutores desta etapa foram denominados tradutores 3 e 4 . As versões retrotraduzidas oriundas desta etapa foram denominadas Versão Retrotraduzida 1 (VRT1) e Versão Retrotraduzida (VRT2).

As versões traduzidas (VT1 e VT2), as versões retrotraduzidas (VRT1 e VRT2), a Versão Consensual em Português 1 do CMDQ (VCP1) e a original foram submetidas a avaliação por um comitê de especialistas. Para esta etapa foram convidados cinco especialistas que deveriam atender aos seguintes critérios: ter conhecimento sobre ergonomia, adaptação e validação de instrumentos e ter domínio da língua portuguesa do Brasil e inglesa.

Os especialistas foram convidados para participar dessa etapa da pesquisa por meio de contato por correio eletrônico. Foi enviado um convite por correio eletrônico explicando sobre os objetivos, os procedimentos da pesquisa e o Termo de 
Consentimento Livre e Esclarecido (TCLE) e o formulário de caracterização sociodemográfica. O TCLE e o formulário de caracterização foram enviados aos especialistas, que deveriam acessá-los por meio de um link restrito do GoogleForms®. As versões VT1, VT2, VRT1, VRT2, VCP1 e a original do CMDQ foram enviadas por meio da uma planilha do programa Microsoft Excel®. O prazo máximo para o retorno dos instrumentos respondidos pelo comitê de especialistas foi de até 15 dias após o envio pelos pesquisadores.

Na planilha do programa Microsoft Excel® os especialistas deveriam analisar cada um dos itens da VT1 e da VT2 comparando com a versão original do CMDQ e selecionar o qual considera mais adequado e compreensível. A avaliação foi realizada por meio de uma escala tipo Likert que varia de 1 a 4 , sendo 1 = item não relevante ou não representativo, $2=$ item necessita de grande revisão para ser representativo; 3 = item necessita de pequena revisão para ser representativo; $4=$ item relevante ou representativo (Rubio et al., 2003; Lynn, 1986). Os especialistas poderiam sugerir alterações nos itens que considerassem necessitar de revisão. A etapa do comitê de especialistas ocorreu de 05 de fevereiro de 2021 a 08 de março de 2021.

Após a avaliação do comitê de especialistas, os pesquisadores realizaram as análises sendo geradas a Versão Consensual em Português 2 do CMDQ (VCP2) e a Versão Consensual em Inglês 1 (VCI1).

A Versão Consensual em Inglês 1 (VCI1) do CMDQ foi enviada no dia 19 de março de 2021, via correio eletrônico, ao Dr. Alan Hedge, o autor da versão original do questionário, para avaliação de possíveis diferenças entre as versões do instrumento. O objetivo dessa etapa foi garantir as equivalências semântica, idiomática, conceitual e cultural do instrumento entre as versões, original e adaptada.

A VCP1 foi submetida ao pré-teste. O objetivo desta etapa foi avaliar a compreensão final da versão adaptada do questionário pela população a que se destina para deteç̧ão de possíveis problemas na redação da versão adaptada do CMDQ.

Para esta etapa foram selecionados 30 participantes (Beaton et al., 2000) de acordo com os seguintes critérios de inclusão: brasileiros, maiores de 18 anos, que atuam como docentes, técnicos administrativos ou funcionários terceirizados do Centro Universitário Maurício de Nassau (UNINASSAU) de Caruaru, Estado de Pernambuco. Foram excluídos os indivíduos que estavam de férias ou em afastamento durante o período da coleta de dados.

Foi enviado um convite para participação da pesquisa informando as pessoas sobre os objetivos, as orientações sobre os procedimentos do estudo e o link do Google Forms ${ }^{\circledR}$ contendo o TCLE. Após aceitar a participar da pesquisa, o participante teve acesso aos seguintes instrumentos: o questionário de caracterização da amostra contendo a Escala Visual Analógica (EVA), a Versão Consensual em Português 1 do CMDQ (VCP1), o questionário de Avaliação da Praticabilidade de Instrumentos (Colucil \& Alexandre, 2009) e o Questionário de dor McGill (Pimenta \& Teixeira, 1996). A EVA e o Questionário de dor McGill não foram utilizados para análises estatísticas na etapa do pré-teste, tais instrumentos foram aplicados com o objetivo de serem utilizados em análise posterior, na etapa de validação, caso a amostra do pré-teste seja incluída na amostra total para validação do CMDQ. A etapa do pré-teste foi realizada de 20 a 22 de abril de 2021.

As etapas de adaptação cultural do CMDQ estão descritas na Figura 1. 
Figura 1. Descrição das etapas do processo de adaptação cultural do Cornell Musculoskeletal Discomfort Questionnaire (CMDQ). Caruaru, Pernambuco, Brasil, 2019-2021.

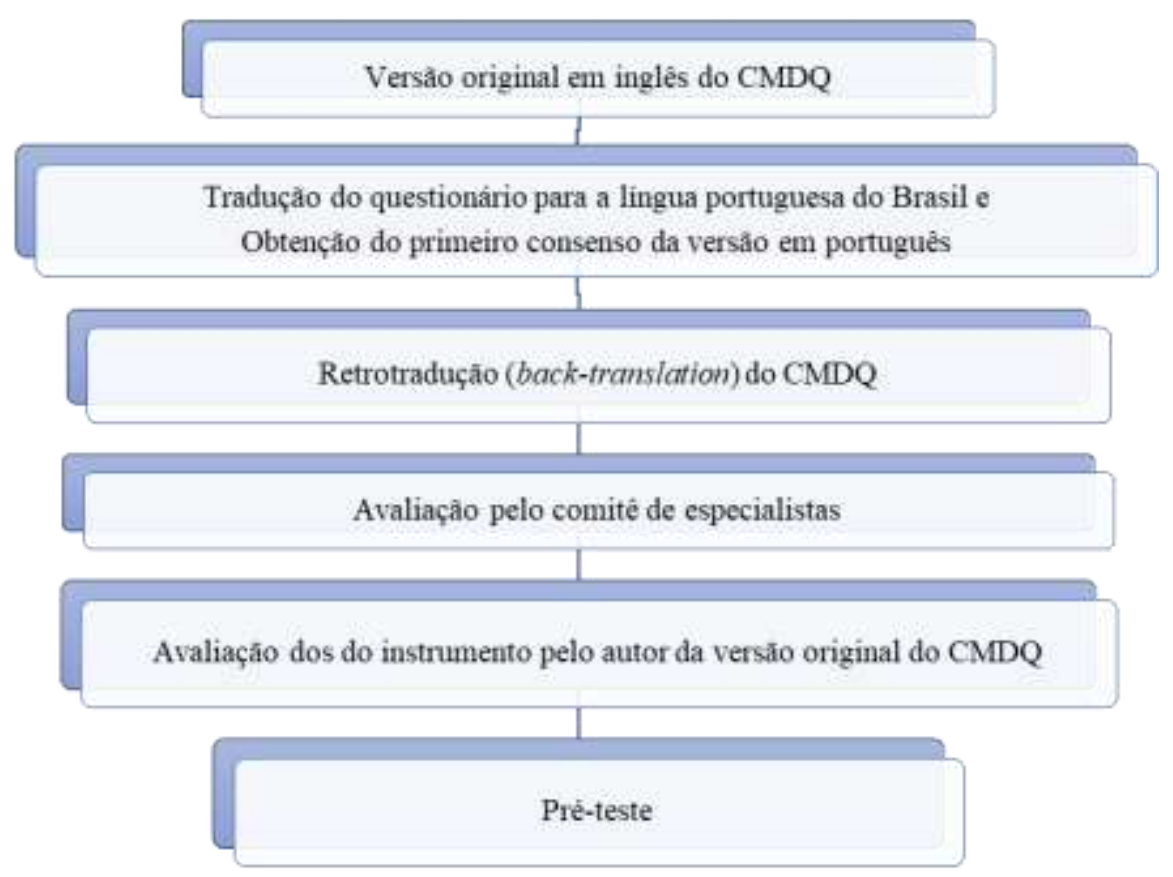

Fonte: Autores.

\subsection{Instrumentos}

\subsubsection{Questionário para a caracterização da amostra}

Os participantes responderam um questionário de dados sociodemográficos, a saber: data de nascimento, sexo, estado civil, profissão, formação e tempo de atuação no trabalho atual.

\subsubsection{Cornell Musculoskeletal Discomfort Questionnaire (CMDQ)}

O questionário Cornell Musculoskeletal Discomfort Questionnaire (CMDQ) foi desenvolvido por pesquisadores na Universidade de Cornell, nos EUA, com a finalidade de uso para pesquisa e não para fins de diagnóstico. O CMDQ tem como objetivo avaliar os sintomas musculoesqueléticos entre a força de trabalho, abordando a frequência, a gravidade e a interferência do trabalho no desconforto músculo esquelético, distribuído em 12 partes do corpo humano (Hedge et al., 1999).

As pontuações do CMDQ podem ser analisadas de quatro maneiras: 1 - Simplesmente contando o número de sintomas por pessoa; 2 - Somando os valores de classificação para cada pessoa; 3 - Ponderando as pontuações de classificação para identificar mais facilmente os problemas mais graves da seguinte forma: Nunca $=0 ; 1$ a 2 vezes por semana = 1.5; 3 a 4 vezes por semana $=3.5$; Todos os dias $=5$; Várias vezes todos os dias $=10$ e 4 - Multiplicando o escore de frequência acima $(0 ; 1,5 ; 3,5 ; 5 ; 10)$ pelo escore de desconforto $(1,2,3)$ pelo escore de interferência $(1,2,3)$ avaliados pelo CMDQ. Quanto maior o escore, maior a frequência e dor musculoesquelética relacionadas ao trabalho (Hedge et al., 1999).

O CMDQ foi adaptado e validado para o idioma turco, persa, espanhol e alemão e identificada boas características psicométricas (Kreuzfeld et al., 2016; Carrasquero, 2015; Afifehzadeh-Kashani et al., 2011; Erdinc et al., 2011). 


\subsubsection{Avaliação da Praticabilidade de Instrumentos}

O questionário de Avaliação da Praticabilidade de Instrumentos foi desenvolvido para avaliar a usabilidade de questionários e o conhecimento das pessoas. O objetivo deste instrumento é mensurar a compreensão e a facilidade dos sujeitos ao responderem o questionário que está sendo adaptado e validado, o CMDQ. Contém três questões com uma escala de resposta de 1 a 5 (discordo totalmente a concordo totalmente) (Pimenta \& Teixeira, 1996).

\subsection{Análise dos dados}

Os dados obtidos por meio da aplicação dos instrumentos foram automaticamente transferidos para uma planilha do programa Microsoft Excel®. Após essa etapa, os dados foram transferidos e analisados por meio do Programa de Software Statistical Package for Social Science (SPSS)® versão 21.0 para cálculo das análises descritivas, de dispersão (desvio-padrão) e as análises psicométricas. O nível de significância adotado para os testes foi de 0,05.

Os dados foram analisados de forma descritiva e inferencial. Primeiramente, as variáveis sociodemográficas da amostra e suas respostas a CMDQ foram analisadas com base em métodos descritivos. As variáveis contínuas foram descritas por meio de média e desvio padrão enquanto as variáveis categóricas foram apresentadas na forma de frequências absolutas e relativas.

Para análise da validade de conteúdo foi calculado o índice de validade de conteúdo (IVC) dos dados oriundos da avaliação do comitê de especialistas. O IVC mensura a proporção ou porcentagem de especialistas que estão em concordância sobre determinados aspectos do questionário e dos itens. Por meio do IVC é possível avaliar cada item individualmente e, posteriormente, o questionário como um todo. O escore do IVC foi calculado por meio da soma de concordância dos itens que marcados com o escore por "3" ou "4" pelos especialistas (Grant \& Davis, 1997). Os itens que receberam pontuação "1" ou "2" deveriam ser revisados ou eliminados. O valor do IVC por item, denominado de Coeficiente de Validade de Conteúdo por item (CVCi), foi calculado por meio das médias das respostas dos especialistas dividido pelo número de especialistas. O valor do CVCi deve ser superior a 0,78 (Polit \& Beck, 2006). Para a verificação da validade do novo instrumento de uma forma geral, o valor do IVC para o escore total do instrumento deve ser de 0,8035 e, preferencialmente, superior a 0,90 (Polit \& Beck, 2006).

A confiabilidade da versão adaptada do CMDQ foi investigada utilizando o alfa de Cronbach sendo considerado valores acima de 0,70 (Aaronson $\mathrm{N}$ et al., 2002).

\section{Resultados e Discussão}

A adaptação cultural do CMDQ seguiu as etapas preconizadas na literatura (Beaton et al., 2007; Beaton et al., 2000; Guillemin et al., 1993). Outros estudos de adaptação de instrumentos desenvolvidos no Brasil adotaram etapas semelhantes (Santos, Nascentes, Freitas, Oliveira \& Castro, 2020; Freitas et al., 2017; Freitas, Caltran, Dantas \& Rossi, 2014; Ferreira, Dantas, Rossi \& Ciol, 2008).

O CMDQ foi submetido a tradução para o português por dois tradutores independentes. Na etapa de síntese das traduções que teve como objetivo obter um consenso entre as versões traduzidas do CMDQ do inglês para o português poucas divergências foram observadas entre as versões. A partir das duas versões traduzidas foi desenvolvida uma versão consensual a qual foi submetida a retrotradução. Neste estudo, optou-se por realizar a retrotradução do questionário antes da etapa do comitê de especialistas conforme a recomendação de Beaton et al., (2007).

As versões traduzidas, a versão consensual em português (VCP1) e as versões retrotraduzidas foram submetidas a avaliação por um comitê composto por cinco especialistas. Dois especialistas recomendaram que os termos "dor passageira" e “dor intensa” fossem substituídos, pois possuem equivalência semântica na língua portuguesa e, além disso, podem induzir a resposta do participante. Um dos especialistas sugeriu alterar os termos "interferiu ligeiramente" por "interferiu levemente" e 
"interferiu substancialmente" por "interferiu consideravelmente". Além disso, os especialistas sugeriram a alteração do termo "pulso" para "punho" devido a nomenclatura correta referente a parte do corpo humano. E, por fim, subtrair o termo coloquial "canela" mantendo somente o termo "parte inferior da perna". Para realizar as alterações sugeridas, os pesquisadores consideraram a avaliação o IVC e CVCi e da pertinência das recomendações dos especialistas. O valor total do IVC do CMDQ foi de 0,78. Sete itens apresentaram CVCi abaixo de 0,78 (variação de 0,68 a 0,76), sendo que a maioria dos itens que os especialistas recomendaram alterações apresentou CVCi abaixo de 0,78, valor recomendado pela literatura (Polit \& Beck, 2006).

A etapa do comitê de especialistas é relevante para a identificação de termos que podem não ser compreendidos pela população alvo, desta forma destaca-se a importância da experiência dos especialistas na temática do questionário (Guillemin et al., 1993). Após a avaliação do comitê de especialistas, os pesquisadores realizaram as análises sendo geradas a Versão Consensual em Português 2 do CMDQ (VCP2) e a Versão Consensual em Inglês 1 (VCI1).

A VCI1 foi enviada ao autor da versão original do CMDQ, o Dr. Alan Hedge, no dia 19 de março de 2021 . No dia 21 de março de 2021, o Dr. Alan Hedge respondeu o correio eletrônico informando que a versão adaptada do instrumento apresentava equivalência com a versão original. Sendo assim, após essa etapa foi mantida a VCI1, não sendo, portanto, realizadas alterações na VCP2. A VCI1 foi renomeada como Versão Final em Inglês (VFI).

O envio da versão retrotraduzida do instrumento para avaliação do autor da versão original é uma etapa recomendada na literatura (Guillemin et al., 1993) com o objetivo de garantir as equivalências semântica, idiomática, conceitual e cultural da versão adaptada do questionário. Os pesquisadores optaram por manter o título do questionário em inglês. A VCP2 foi denominada de Versão Adaptada Final do CMDQ.

No Quadro 1, estão apresentadas a versão original, as versões traduzidas VT1 e VT2, a VPC1 e a versão final adaptada do CMDQ. Na Tabela 1, estão apresentadas o resultado da validade de conteúdo do CMDQ. 
Quadro 1. Resultados da etapa de tradução do Cornell Musculoskeletal Discomfort Question (CMDQ). Caruaru, Pernambuco, Brasil, 2019.

\begin{tabular}{|c|c|c|c|c|}
\hline Versão original do CMDQ & Versão Tradutor 1 (VT1) & Versão Tradutor 2 (VT2) & $\begin{array}{l}\text { Versão em Português } \\
\text { Consensual } 1 \text { (VPC1) }\end{array}$ & $\begin{array}{c}\text { Versão Adaptada Final do } \\
\text { CMDQ }\end{array}$ \\
\hline $\begin{array}{l}\text { Cornell Musculoskeletal } \\
\text { Discomfort Question (CMDQ) }\end{array}$ & $\begin{array}{l}\text { Questionário de desconforto } \\
\text { musculoesquelético de Cornell } \\
\text { (QDMC) }\end{array}$ & $\begin{array}{l}\text { Questionário Cornell de } \\
\text { Desconforto } \\
\text { Musculoesquelético (QCDM) }\end{array}$ & $\begin{array}{l}\text { Questionário Cornell de } \\
\text { Desconforto } \\
\text { Musculoesquelético (QCDM) }\end{array}$ & $\begin{array}{l}\text { Questionário Cornell de } \\
\text { Desconforto } \\
\text { Musculoesquelético (QCDM) }\end{array}$ \\
\hline $\begin{array}{l}\text { The diagram below shows the } \\
\text { approximate position of the } \\
\text { body parts referred to in the } \\
\text { questionnaire. Please answer by } \\
\text { marking the appropriate box. }\end{array}$ & $\begin{array}{l}\text { O diagrama abaixo mostra a } \\
\text { posição aproximada das partes } \\
\text { do corpo mencionadas no } \\
\text { questionário. Por favor, } \\
\text { responda marcando a } \\
\text { alternativa apropriada. }\end{array}$ & $\begin{array}{l}\text { O diagrama abaixo mostra a } \\
\text { posição aproximada das partes } \\
\text { do corpo referidas no } \\
\text { questionário. Por favor, } \\
\text { responda marcando a caixa } \\
\text { apropriada. }\end{array}$ & $\begin{array}{l}\text { O diagrama abaixo mostra a } \\
\text { posição aproximada das partes } \\
\text { do corpo mencionadas no } \\
\text { questionário. Por favor, } \\
\text { responda assinalando a } \\
\text { alternativa apropriada. }\end{array}$ & $\begin{array}{l}\text { O diagrama abaixo mostra a } \\
\text { posição aproximada das partes } \\
\text { do corpo mencionadas no } \\
\text { questionário. Por favor, } \\
\text { responda assinalando a } \\
\text { alternativa apropriada. }\end{array}$ \\
\hline $\begin{array}{l}\text { During the last work week how } \\
\text { often did you experience ache, } \\
\text { pain, discomfort in: } \\
\text { Never } \\
1-2 \text { times last week } \\
\text { 3-4 times last week } \\
\text { Once every day } \\
\text { Several times every day }\end{array}$ & $\begin{array}{l}\text { Durante a última semana de } \\
\text { trabalho, com que frequência } \\
\text { você sentiu dor, desconforto: } \\
\text { Nunca } \\
\text { 1-2 vezes na última semana } \\
\text { 3-4 vezes na última semana } \\
\text { Uma vez todos os dias } \\
\text { Várias vezes todos os dias }\end{array}$ & $\begin{array}{l}\text { Durante a última semana de } \\
\text { trabalho, com que frequência } \\
\text { você sentiu dor ou desconforto: } \\
\text { Nunca } \\
1-2 \text { vezes na última semana } \\
3-4 \text { vezes na última semana } \\
\text { Uma vez por dia } \\
\text { Várias vezes por dia }\end{array}$ & $\begin{array}{l}\text { Durante a última semana de } \\
\text { trabalho, com que frequência } \\
\text { você sentiu uma dor } \\
\text { passageira, dor intensa, } \\
\text { desconforto em: } \\
1 \text { - } 2 \text { vezes na última semana } \\
3 \text { - } 4 \text { vezes na última semana } \\
\text { Uma vez por dia } \\
\text { Várias vezes por dia }\end{array}$ & $\begin{array}{l}\text { Durante a última semana de } \\
\text { trabalho, com que frequência } \\
\text { você sentiu dor e desconforto } \\
\text { em: } \\
1-2 \text { vezes na última semana } \\
3-4 \text { vezes na última semana } \\
\text { Uma vez por dia } \\
\text { Várias vezes por dia }\end{array}$ \\
\hline $\begin{array}{l}\text { If you experienced ache, pain } \\
\text { discomfort, how uncomfortable } \\
\text { was this? } \\
\text { Slightly uncomfortable } \\
\text { Moderately uncomfortable } \\
\text { Very uncomfortable }\end{array}$ & $\begin{array}{l}\text { Se você sentiu dor, desconforto, } \\
\text { quão desconfortável foi isso? } \\
\text { Um pouco desconfortável } \\
\text { Moderadamente desconfortável } \\
\text { Muito desconfortável }\end{array}$ & $\begin{array}{l}\text { Se você sentiu dor ou } \\
\text { desconforto, o quão } \\
\text { desconfortável foi? } \\
\text { Levemente desconfortável } \\
\text { Moderadamente desconfortável } \\
\text { Muito desconfortável }\end{array}$ & $\begin{array}{l}\text { Se você sentiu dor passageira, } \\
\text { dor intensa, desconforto quão } \\
\text { desconfortável foi isso? } \\
\text { Levemente desconfortável } \\
\text { Moderadamente desconfortável } \\
\text { Muito desconfortável }\end{array}$ & $\begin{array}{l}\text { Se você sentiu dor e } \\
\text { desconforto quão } \\
\text { desconfortável foi isso? } \\
\text { Levemente desconfortável } \\
\text { Moderadamente desconfortável } \\
\text { Muito desconfortável }\end{array}$ \\
\hline $\begin{array}{l}\text { If you experienced ache, pain, } \\
\text { discomfort, did this interfere } \\
\text { with your ability to work? } \\
\text { Not at all } \\
\text { Slightly interfered } \\
\text { Substantially interfered }\end{array}$ & $\begin{array}{l}\text { Se você sentiu dor, desconforto, } \\
\text { isso interferiu na sua } \\
\text { capacidade de trabalhar? } \\
\text { De modo nenhum } \\
\text { Interferiu ligeiramente } \\
\text { Interferiu substancialmente }\end{array}$ & $\begin{array}{l}\text { Se você sentiu dor ou } \\
\text { desconforto, isso interferiu na } \\
\text { sua habilidade de trabalhar? } \\
\text { Nem um pouco } \\
\text { Interferiu levemente } \\
\text { Interferiu substancialmente }\end{array}$ & $\begin{array}{l}\text { Se você sentiu dor passageira, } \\
\text { dor intensa, desconforto isso } \\
\text { interferiu na sua capacidade de } \\
\text { trabalhar? } \\
\text { Nem um pouco } \\
\text { Interferiu ligeiramente } \\
\text { Interferiu substancialmente }\end{array}$ & $\begin{array}{l}\text { Se você sentiu dor e } \\
\text { desconforto isso interferiu na } \\
\text { sua capacidade de trabalhar? } \\
\text { Nem um pouco } \\
\text { Interferiu levemente } \\
\text { Interferiu consideravelmente }\end{array}$ \\
\hline Neck & Pescoço & Pescoço & Pescoço & Pescoço \\
\hline
\end{tabular}




\begin{tabular}{|c|c|c|c|c|}
\hline Shoulder & Ombro & Ombro & Ombro & Ombro \\
\hline Upper Back & Parte superior das costas & Parte superior das costas & Parte superior das costas & Parte superior das costas \\
\hline Upper Arm & Parte superior do braço & Parte superior do braço & Parte superior do braço & Parte superior do braço \\
\hline Lower Back & Parte inferior das costas & Parte inferior das costas & Parte inferior das costas & Parte inferior das costas \\
\hline Forearm & Antebraço & Antebraço & Antebraço & Antebraço \\
\hline Wrist & Pulso & Pulso & Pulso & Punho \\
\hline Hip/Buttocks & Quadril/Nádegas & Quadril/Nádegas & Quadril/Nádegas & Quadril/Nádegas \\
\hline Thigh & Coxa & Coxa & Coxa & Coxa \\
\hline Knee & Joelho & Joelho & Joelho & Joelho \\
\hline Lower Leg & Canela & Parte inferior da perna & Parte inferior da perna (Canela) & Parte inferior da perna \\
\hline Foot & Pé & Pé & Pé & Pé \\
\hline (Right) & (Direita) & (Direito) & (Direito) & (Direito) \\
\hline (Left) & (Esquerda) & (Esquerdo) & (Esquerdo) & (Esquerdo) \\
\hline
\end{tabular}

Nota: todas as alterações realizadas estão destacadas em negrito. Fonte: Autores; 
Tabela 1 - Resultados da validade de conteúdo da Versão em Português Consensual 1 do CMDQ pelo Comitê de Especialistas. Caruaru, Pernambuco, Brasil, 2021.

\begin{tabular}{|c|c|c|}
\hline Itens & \% concordância ${ }^{a}$ & $\mathrm{CVCi}^{\mathrm{b}}$ \\
\hline $\begin{array}{l}\text { O diagrama abaixo mostra a posição aproximada das partes do } \\
\text { corpo mencionadas no questionário. Por favor, responda } \\
\text { assinalando a alternativa apropriada. }\end{array}$ & 80 & 0,80 \\
\hline $\begin{array}{l}\text { Durante a última semana de trabalho, com que frequência você } \\
\text { sentiu uma dor passageira, dor intensa, desconforto em: }\end{array}$ & 100 & 0,68 \\
\hline Nunca & 80 & 0,80 \\
\hline $1-2$ vezes na última semana & 80 & 0,80 \\
\hline $3-4$ vezes na última semana & 100 & 0,80 \\
\hline Uma vez por dia & 100 & 0,80 \\
\hline Várias vezes por dia & 100 & 0,80 \\
\hline $\begin{array}{l}\text { Se você sentiu dor passageira, dor intensa, desconforto quão } \\
\text { desconfortável foi isso? }\end{array}$ & 100 & 0,76 \\
\hline Levemente desconfortável & 100 & 0,80 \\
\hline Moderadamente desconfortável & 100 & 0,80 \\
\hline Muito desconfortável & 100 & 0,80 \\
\hline $\begin{array}{l}\text { Se você sentiu dor passageira, dor intensa, desconforto isso } \\
\text { interferiu na sua capacidade de trabalhar? } \\
\text { Nem um pouco }\end{array}$ & $\begin{array}{l}100 \\
100\end{array}$ & $\begin{array}{l}0,76 \\
0,80\end{array}$ \\
\hline Interferiu ligeiramente & 100 & 0,72 \\
\hline Interferiu substancialmente & 100 & 0,76 \\
\hline Pescoço & 100 & 0,80 \\
\hline Ombro & 100 & 0,80 \\
\hline Parte superior das costas & 100 & 0,80 \\
\hline Parte superior do braço & 100 & 0,80 \\
\hline Parte inferior das costas & 100 & 0,76 \\
\hline Antebraço & 100 & 0,76 \\
\hline Pulso & 100 & 0,76 \\
\hline Quadril/Nádegas & 100 & 0,80 \\
\hline Coxa & 100 & 0,80 \\
\hline Joelho & 100 & 0,80 \\
\hline Parte inferior da perna ("Canela") & 100 & 0,80 \\
\hline Pé & 100 & 0,80 \\
\hline (Direito) & 100 & 0,80 \\
\hline (Esquerdo) & 100 & 0,80 \\
\hline
\end{tabular}

aPorcentagem de concordância entre os especialistas; ${ }^{\text {b} C o e f i c i e n t e ~ d e ~ V a l i d a d e ~ d e ~ C o n t e u ́ d o ~ p o r ~ i t e m . ~ F o n t e: ~ A u t o r e s . ~}$

Participaram da etapa do pré-teste 30 sujeitos. A amostra foi dividida igualmente entre os sexos. A média de idade foi de 32,8 anos $(\mathrm{DP}=8,0)$. A maioria da raça branca $(56,7 \%)$ e casado $(56,7 \%)$. Todos os participantes têm nível superior 
completo com média de anos de estudo de 19,3 anos ( $\mathrm{DP}=8,9) .93,3 \%$ eram docentes na instituição de ensino superior. 60,0\% relataram que trabalham a maior parte do tempo na posição sentado (Tabela 2).

Tabela 2. Distribuição dos participantes do pré-teste segundo as características sociodemográficas $(\mathrm{n}=30)$. Caruaru, Pernambuco, Brasil, 2021.

\begin{tabular}{|c|c|c|c|c|c|c|}
\hline Variáveis & $\mathbf{N}$ & $(\%)$ & Média & $(\mathbf{D P})^{\mathrm{a}}$ & Mediana & (Intervalo) \\
\hline \multicolumn{7}{|l|}{ Sexo } \\
\hline Feminino & 15 & $(50)$ & & & & \\
\hline Masculino & 15 & $(50)$ & & & & \\
\hline Idade & 30 & & 32,8 & $(8,0)$ & 32,1 & $(25,8-45,9)$ \\
\hline \multicolumn{7}{|l|}{ Raça } \\
\hline Branca & 17 & $(56,7)$ & & & & \\
\hline Parda & 11 & $(36,7)$ & & & & \\
\hline Amarela & 2 & $(6,7)$ & & & & \\
\hline \multicolumn{7}{|l|}{ Estado Civil } \\
\hline Casado(a) & 17 & $(56,7)$ & & & & \\
\hline Solteiro(a) & 10 & $(33,3)$ & & & & \\
\hline Separado(a) & 3 & $(10,0)$ & & & & \\
\hline \multicolumn{7}{|l|}{ Titulação } \\
\hline Superior completo & 30 & $(100)$ & & & & \\
\hline Anos de estudo & 27 & & 19,3 & $(8,9)$ & 20 & $(5-35)$ \\
\hline \multicolumn{7}{|l|}{ Cargo } \\
\hline Docente & 28 & $(93,3)$ & & & & \\
\hline Funcionário terceirizado & 1 & $(3,3)$ & & & & \\
\hline Técnico administrativo & 1 & $(3,3)$ & & & & \\
\hline \multicolumn{7}{|l|}{ Posição trabalho } \\
\hline Sentado(a) & 18 & $(60,0)$ & & & & \\
\hline Em pé & 12 & $(40,0)$ & & & & \\
\hline
\end{tabular}

aDesvio-padrão. Fonte: Autores.

$\mathrm{Na}$ avaliação da praticabilidade da versão adaptada do CMDQ, no tocante ao entendimento das instruções do questionário todos os participantes concordaram totalmente que as instruções são de fácil entendimento, sendo que 93,3\% dos participantes concordaram totalmente que as questões são de fácil entendimento e todos os participantes consideraram fácil assinalar as respostas do questionário (Tabela 3).

Tabela 3. Avaliação da praticabilidade da versão adaptada do CMDQ. Caruaru, Pernambuco, Brasil, 2021.

\begin{tabular}{lccccc}
\hline Questões & $\begin{array}{c}\text { Discordo } \\
\text { totalmente } \\
\mathbf{N}(\%)\end{array}$ & $\begin{array}{c}\text { Discordo } \\
\text { parcialmente } \\
\mathbf{N}(\%)\end{array}$ & $\begin{array}{c}\text { Não tenho } \\
\text { Opinião } \\
\mathbf{N}(\%)\end{array}$ & $\begin{array}{c}\text { Concordo } \\
\text { parcialmente } \\
\mathbf{N}(\%)\end{array}$ & $\begin{array}{c}\text { Concordo } \\
\text { totalmente } \\
\mathbf{N}(\%)\end{array}$ \\
\hline $\begin{array}{l}1-\text { Eu achei fácil } \\
\text { entender as instruções } \\
\text { do questionário }\end{array}$ & $0(0)$ & $0(0)$ & $0(0)$ & $0(0)$ & $30(100)$ \\
$2-$ Eu achei fácil & $0(0)$ & $0(0)$ & $0(0)$ & $2(6,7)$ \\
$\begin{array}{l}\text { entender as questões } \\
\text { do questionário }\end{array}$ & & & & $28(93,3)$ \\
$3 \quad-$ Eu achei fácil & $0(0)$ & $0(0)$ & $0(0)$ & $0(0)$ & $30(100)$ \\
$\begin{array}{l}\text { assinalar as respostas } \\
\text { do questionário }\end{array}$ & & & & \\
\hline
\end{tabular}


A confiabilidade da versão adaptada do CMDQ na etapa do pré-teste foi avaliada por meio do alfa de Cronbach sendo identificado um valor de 0,84 para o escore total. Resultados semelhantes foram identificados na versão validada final do CMDQ para o idioma espanhol e alemão (Kreuzfeld et al., 2016; Carrasquero, 2015). O valor do alfa de Cronbach é fortemente influenciado pelo número amostral, no entanto mesmo com uma amostra reduzida foi identificado um valor de alfa de Cronbach satisfatório no pré-teste do CMDQ (Charter, 2003).

\section{Conclusão}

A etapa adaptação cultural e de avaliação da validade de conteúdo do CMDQ foi finalizada obtendo-se resultados relevantes. O processo de validação do CMDQ para o Brasil está em etapa de finalização para que, assim, a versão final do instrumento possa ser administrada na população brasileira. Destaca-se que no futuro, após a validação do CMDQ, será possível utilizá-lo em estudos futuros para avaliar sintomas musculoesqueléticos relacionados ao trabalho na população brasileira.

\section{Referências}

Aaronson, N. et al. (2002). Scientific Advisory Committee of medical Outcomes Trust. Assessing health status and quality of life instruments: attributes and review criteria. Quality of Life Research, 11, 193-205.

Abrahão, J. I. (2000). Reestruturação Produtiva e Variabilidade do Trabalho: Uma Abordagem da Ergonomia. Psicologia: Teoria e Pesquisa 16(1), 49-54.

Afifehzadeh-Kashani, H., Choobineh, A., Bakand, S., Gohari, M. R., Abbastabar, H. \& Moshtaghi, P. (2011). Validity and reliability of farsi version of Cornell Musculoskeletal Discomfort Questionnaire (CMDQ). Iran Occupational Health, 7(4), 69-75.

Barros, E. N. C. \& Alexandre, N. M. C. (2003). Cross-cultural adaptation of the Nordic musculoskeletal questionnaire. International Nursing Review, 50, 101108.

Beaton, D., Bombardier, C., Guillemin, F., \& Ferraz, M. B. (2007). Recommendations for the crosscultural adaptation of the DASH \& Quick. DASH outcome measures. Institute for Work \& Health.

Beaton, D., Bombardier, C., Guillemin, F., \& Ferraz, M. B. (2000). Guidelines for the process of cross-cultural adaptation of self-report measures. Spine, 25 (24), 3186-3191.

Carrasquero, E. E. C. (2015). Adaptación y validación española del instrumento de percepción Cornell musculoskeletal discomfort questionnaires (CMDQ). Desarrollo Gerencial, 7(2), 36-46.

Carvalho, A. J. F. P. \& Alexandre, N. M. C. (2006). Sintomas osteomusculares em professores do Ensino Fundamental. Brazilian Journal of Physical Therapy, $10(1), 35-41$.

Charter, R. A. (2003). Study samples are too small to produce sufficiently precise reliability coefficients. The Journal of General Psychology, 130(2),117-129.

ColuciI, M. Z. O. \& Alexandre, N. M. C. (2009). Development of a questionnaire to evaluate the usability of assessment instruments. Revista Enfermagem UERJ, 17(3), 378-832.

Erdinc, O., Hot, K. \& Ozkaya, M. (2011). Turkish version of the Cornell Musculoskeletal Discomfort Questionnaire: Cross cultural adaptation and validation. Work, 39(3), 251-260.

Ferreira, E., Dantas, R. A. S., Rossi, L. A. \& Ciol, M. A. (2008). The cultural adaptation and validation of the Burn Specific Health Scale Revised (BSHS-R): version for Brazilian burn victims. Burns, 34(7), 994-1001

Freitas, N. O., Forero, C. G., Alonso, J., Caltran, M. P., Dantas, R. A. S., Farina, J. A. \& Rossi, L. A. (2017). Cross-cultural adaptation and validation of the Social Comfort Questionnaire for Brazilian adult survivors of burns. Quality of Life Research, 26,205-211.

Freitas, N. O., Caltran, M. P., Dantas, R. A. S., Rossi, L. A. (2014). Translation and cultural adaptation of the Perceived Stigmatization Questionnaire for burn victims in Brazil. Revista da Escola de Enfermagem da USP, 48, 25-33.

Guillemin, F., Bombardier, C. \& Beaton, D. E. (1993). Crosscultural adaptation of health - related quality of life measures: literature review and proposed guidelines. Journal of Clinical Epidemiology, 46 (12), 1427-1432.

Grant, J. S. \& Davis, L. L. (1997). Selection and use of content experts for instrument development. Research in Nursing \& Health, $20(3), 269-274$.

Hedge, A., Morimoto, S, \& McCrobie, D. (1999). Effects of keyboard tray geometry on upper body posture and comfort. Ergonomics, 42 (10), $1333-1349$.

Kreuzfeld, S., Seibt, R., Kumar, M., Rieger, A. \& Stoll, R. (2016). German version of the Cornell Musculoskeletal Discomfort Questionnaire (CMDQ): Translation and validation. Journal of Occupational Medicine and Toxicology, 11:13. 
Research, Society and Development, v. 10, n. 12, e428101220412, 2021

(CC BY 4.0) | ISSN 2525-3409 | DOI: http://dx.doi.org/10.33448/rsd-v10i12.20412

Kuorinka, I. et al. (1987). Standardised Nordic Questionnaires for the Analysis of Musculoskeletal Symptoms. Applied Ergonomics, 18 , $233-237$.

Lynn, M. R. (1986). Determination and quantification of content validity. Nursing Research, 35(6), 382-385.

Monteiro, M. A. M. (2009). Importância da ergonomia na saúde dos funcionários de unidades de alimentação e nutrição. Revista Baiana, 33(3), 416-427.

Mota, I. L., Quadros Júnior, M. C., Munaro, H. L. R. \& Vilela, A. B. (2014). Sintomas osteomusculares de servidores de uma universidade pública brasileira: um estudo ergonômico. Revista Brasileira em Promoção da Saúde, 27(3), 341-348.

Pimenta, C. A. M \& Teixeira, M. J. (1996). Questionário de dor McGill: proposta de adaptação para a língua portuguesa. Revista da Escola de Enfermagem da USP, 30 (3), 473-483.

Polit, D. F. \& Beck, C. T. (2006). The content validity index: are you sure you know what's being reported? Critique and recommendations, Research in Nursing \& Health, 29(5), 489-497.

Rubio, D. M., Berg-Weger, M., Tebb, S. S., Lee, S. \& Rauch, S. (2003). Objectifying content validity: conducting a content validity study in social work research. Social Work Research, 27(2), 94-105.

Santos, V. L. C. G., Nascentes, C. C., Freitas, N. O., Oliveira, M. W. \& Castro, D. L. V. (2020). Cultural Adaptation and Validation of the Ostomy Adjustment Inventory-23 for Brazil. Wound Management \& Prevention, 66, 32-40.

Serranheira, F. et al. (2004). Da saúde e segurança do trabalho à saúde e segurança dos trabalhadores: um (ainda) longo caminho a percorrer: $2{ }^{a}{ }^{\text {parte: }}$ uma perspectiva da ergonomia no contexto da saúde e segurança do trabalho (SST). Segurança. Ano XLIX: 2009a; 189:18-23.

Vitta, A. De,Bertaglia, R. S. \& Padovani, C. R. (2008). Efeitos de dois procedimentos educacionais sobre os sintomas musculoesqueléticos em trabalhadores administrativos. Brazilian Journal of Physical Therapy, 12 (1), 20-25. 